\title{
Übergang in und Anpassung an den Ruhestand als Herausforderung aus psychologischer Perspektive
}

\author{
Antje Schmitt
}

Online publiziert: 12 . Juli 2018

(C) Der/die Autor(en) 2018

Zusammenfassung Anhand von theoretischen Ansätzen wird dargestellt, warum der Ruhestandsprozess als Herausforderung für den Einzelnen gilt. Zudem werden empirische Erkenntnisse der zentralen Faktoren zusammengefasst, die den Übergang und eine erfolgreiche Anpassung an die neue Lebensphase determinieren. $\mathrm{Zu}$ empirisch bisher bestätigten Faktoren gehören mitunter das individuelle Gesundheitsniveau, finanzielle Ressourcen, das Ausmaß der Freiwilligkeit des Übergangs, Antizipation und Planung des Ruhestands, die soziale Eingebundenheit und Unterstützung, das Engagement in sinnhaften und erfüllenden Aktivitäten im Ruhestand sowie der Erhalt der Alltagsstruktur. Abschließend wird die Notwendigkeit der Verzahnung von praktischen Ansätzen mit bestehender empirischer Evidenz betont.

Schlüsselwörter Ruhestand $\cdot$ Lebensabschnitt $\cdot$ Anpassung $\cdot$ Ressourcen

\section{The transition and adjustment to retirement from a psychological perspective}

Abstract Based on theoretical approaches, it is shown why the retirement process may be considered a challenge for the individual. Moreover, empirical findings regarding the central factors that determine the transition and successful adjustment to this stage of life have been summarized. Empirically validated factors include individual health levels, financial resources, information on whether the transition is voluntary or not, the anticipation and planning of retirement, social integration and support, engagement in meaningful and fulfilling activities during retirement,

Dr. A. Schmitt $(\bowtie)$

Department of Psychology, University of Groningen, Grote Kruisstraat 2/1, 9712 TS Groningen,

Niederlande

E-Mail: a.schmitt@rug.nl 
and the preservation of a day-to-day structure. Finally, the importance of integrating practical approaches with existing empirical evidence is emphasized.

Keywords Retirement - Life stage $\cdot$ Adjustment $\cdot$ Resources

\section{Einleitung}

Der demographische Wandel in den westlichen Industriegesellschaften impliziert eine steigende Lebenserwartung des Einzelnen bei gleichzeitig stagnierender Geburtenrate. Daraus ergibt sich der Trend einer kontinuierlichen Zunahme von Personen im höheren Lebensalter ( $a b$ dem 65. Lebensjahr). Mehr und mehr Menschen sind mit dem Thema Übergang in den Ruhestand konfrontiert. Vor dem Hintergrund der demographischen Entwicklung und zur Sicherung der Finanzierbarkeit des Rentensystems beobachtet man in den letzten Jahrzehnten den Trend einer schrittweisen Anhebung des Renteneintrittsalters (Statistisches Bundesamt, 2006; Tesch-Römer et al. 2006), sodass die Erwerbsbeteiligung älterer Menschen in den vergangenen Jahren deutlich angestiegen ist. Zunehmend mehr Menschen sind auf vielfältige Weise auch im Ruhestand auf dem Arbeitsmarkt aktiv (Wöhrmann et al. 2017), und der Übergang in den Ruhestand wird heutzutage zunehmend flexibel gestaltet (z. B. durch Maßnahmen wie Altersteilzeitregelungen) (Hübner 2016).

Der Ausstieg aus dem Erwerbsleben und die Anpassung an die Phase des Ruhestands sind in den letzten beiden Jahrzehnten in der gesundheits-, arbeits- und entwicklungspsychologischen Forschung auf ein wachsendes Interesse gestoßen (Hübner 2016; Wang 2012; Wang und Shi 2014). Ich werde im Folgenden zunächst die Begriffe des Übergangs und der Anpassung an den Ruhestand definieren und anschließend erläutern, warum sie als psychologische Herausforderungen gelten. Ferner werden verschiedene Faktoren dargestellt, die den Übergang in den Ruhestand und die Anpassung daran auf Basis theoretischer Annahmen sowie empirischer Erkenntnisse determinieren.

\section{Der Ruhestand: Definition und Konzeptualisierung}

Es existiert keine präzise und allgemeingültige Definition des Begriffs des Ruhestands (Wang und Shi 2014). Denton und Spencer (2009) stellen eine Übersicht acht verschiedener in der Psychologie existierender Definitionen und Indikatoren des Ruhestands dar, die gelegentlich kombiniert verwendet werden. Ruhestand bezieht sich auf das Ausscheiden aus der Erwerbsarbeit, und die Nichtteilnahme am Arbeitsleben wird häufig ergänzt um weitere Eigenschaften wie der mentalen sowie verhaltensbezogenen Distanzierung vom Arbeitsleben oder der Bezug von monetären Mitteln aus einer eigenen früheren Arbeitstätigkeit. Aufgrund der Vielfalt möglicher Definitionen ergibt sich gleichzeitig eine fehlende präzise Messung des Ruhestands. Häufig werden Personen im Selbstbericht danach gefragt, ob ein Übergang in den Ruhestand erfolgt ist (Denton und Spencer 2009). 
Thematisch lässt sich die Beschäftigung mit dem Konzept des Ruhestands innerhalb der Psychologie in verschiedenen grundlageorientierten und anwendungsbezogenen Disziplinen verorten. Die theoretische Basis liegt vorrangig in Ansätzen der Entwicklungs- bzw. Lebensspannenpsychologie. Nach dem Konzept der Entwicklungsaufgaben (Havighurst 1961), demzufolge es im Laufe der Entwicklung des Menschen von der Kindheit bis ins hohe Erwachsenenalter Aufgaben und Anforderungen zu bewältigen gibt, die an Menschen herangetragen werden, kann der Ruhestand und die Anpassung an die damit einhergehenden Veränderungen sowie das Finden neuer Rollen und Aktivitätsbereiche als Entwicklungsaufgabe betrachtet werden. Neuere Konzepte der Lebensspannenpsychologie thematisieren die Frage, wie erfolgreiches Altern gelingen kann (Baltes und Baltes 1990; Brandtstädter und Renner 1990; Heckhausen et al. 2010). Erfolgreiches Altern ist die geglückte Anpassung an die sozialen, biologischen und psychischen Prozesse, die mit dem zunehmenden Alter auftreten (Baltes und Baltes 1990; Hübner 2016; Zacher 2015). Ziel ist die Maximierung von Entwicklungsgewinnen (z. B. Optimierung erworbener Fertigkeiten, Erhalt des persönlichen Wohlbefindens durch Anpassung von Lebenszielen an individuelle Gegebenheiten, wie die eigene gesundheitliche und finanzielle Situation) und die Minimierung von funktionalen Einschränkungen im Alter (u.a. körperliche Abbauprozesse, Abnahme der fluiden Intelligenz, Veränderung von Muskulatur, Herz-Kreislauf-System, Energiestoffwechsel und Hormonhaushalt) (Baltes und Baltes 1990; Hübner 2016). Theoretische Ansätze der Entwicklungs- bzw., Lebensspannenpsychologie werden nun vielfältig unter anderem von der Arbeits- und Organisationspsychologie als angewandte Disziplin aufgegriffen, wenn es z.B. um die Frage geht, wie berufliche Laufbahnentwicklungskonzepte, Coaching und Beratung per se gestaltet werden können, um eine erfolgreiche Entwicklung für den Einzelnen im Ruhestand zu gewährleisten.

\section{3 Übergang in den Ruhestand als Entwicklungsprozess}

Der Übergang beschreibt die Veränderung im Lebensstatus einer Person über die Zeit. Man befindet sich z.B. im Arbeitsleben, und durch das diskrete Ereignis des Übergangs in den Ruhestand erreicht man den neuen Status oder chronologischen Abschnitt (Bliese et al. 2017; Wang 2007). Wann genau der Übergang stattfindet und wie die Veränderung des Lebensstatus empfunden wird, ist von verschiedenen Beschreibungsebenen abhängig (Bliese et al. 2017; Fisher et al. 2016; Zacher 2017). So kann der Übergang abrupt erfolgen oder graduell, geplant oder ungeplant, freiwillig oder unfreiwillig stattfinden. Wie der Übergang wahrgenommen wird, hat wiederum Konsequenzen für die Anpassung an den neuen Lebensabschnitt.

Die Anpassung an den Ruhestand meint die Qualität des Verlaufs nach erfolgtem Übergang. Ziel ist es, eine gelungene Anpassung zu erreichen, sodass die neue Lebensphase akzeptiert und von der betroffenen Person in die eigene Identität integriert werden kann (Pinquart und Schindler 2007; Van Solinge und Henkens 2008; Wang und Shi 2014). Um Aussagen über die Qualität der Anpassung zu treffen, können wiederum vielfältige Indikatoren herangezogen werden. In der psychologischen Literatur werden häufig verschiedene Aspekte von Gesundheit bzw. Wohlbefinden 
erfasst. Gesundheit wird als mehrdimensionales Konstrukt betrachtet (Hübner 2016), bestehend aus körperlichen, psychischen und sozialen Aspekten (Lippke und Renneberg 2006; WHO 2017). In einzelnen Studien wird zudem auch das finanzielle Wohlbefinden herangezogen, in der Regel erfasst über die Zufriedenheit mit der finanziellen Situation oder auch über das Haushaltseinkommen.

Der Übergang in den Ruhestand steht am Ende eines individuellen Entwicklungsprozesses. Shultz und Wang (2011) stellen in ihrem Prozessmodell des Ruhestands dar, dass der Ruhestand in der Regel bereits einige Jahre vor dem eigentlichen Übergang antizipiert wird und Planungsprozesse eintreten (Shultz und Wang 2011; Wang und Shi 2014). Später entscheidet man sich für den konkreten Ausstieg aus der Erwerbstätigkeit, was gefolgt ist vom anschließenden Übergang und der Anpassung.

\section{Determinanten des Übergangs in den Ruhestand}

Die Terminierung des Übergangs in den Ruhestand wird beeinflusst durch kontextuelle, arbeitsbezogene und individuelle Faktoren sowie deren Zusammenspiel. Kontextuelle Faktoren beziehen sich z. B. auf Möglichkeiten, die durch die staatliche Renten- und Arbeitsmarktpolitik bestehen (u.a. Umfang der sozialen Absicherung in der Rente). So ist z. B. ein früherer Übergang wahrscheinlicher, je besser die sozialstaatlichen Unterstützungssysteme ausgebaut sind (Fisher et al. 2016; Wang und Shi 2014). Ferner spielen organisationale Einflussfaktoren (z. B. altersgerechte Personalstrategie wie Ermöglichung eines gleitenden Übergangs in den Ruhestand) eine Rolle für die Terminierung des Ruhestands. Zu den arbeitsbezogenen Faktoren gehören insbesondere das Ausmaß der Verbundenheit und Identifizierung mit der beruflichen Tätigkeit und mit dem Arbeitgeber sowie das Belastungserleben in der Arbeit. Je stärker die Verbundenheit, desto eher entscheidet man sich dafür, später in den Ruhestand zu gehen oder nach Erreichen der Altersgrenze weiterzuarbeiten, z.B. in geringerem zeitlichem Umfang. Ein früherer Übergang wird eher dann in Erwägung gezogen, wenn die berufliche Tätigkeit sehr belastend ist.

Eine Vielzahl individueller Faktoren beeinflusst die Terminierung des Übergangs (Fisher et al. 2016). So führt ein geringes Gesundheitsniveau in der Regel dazu, dass man die Erwerbstätigkeit früher beendet, da man die Arbeit nicht mehr bewältigen kann. Zugleich kann jedoch auch ein sehr guter Gesundheitszustand mit einem frühzeitigen Ausscheiden aus dem Beruf verbunden sein (de Wind et al. 2013; Fisher et al. 2016), wenn die Personen ihren Ruhestand genießen und aktiv sein wollen, solange die Gesundheit dies noch zulässt. Der Effekt einer positiven Korrelation zwischen sehr guter Gesundheit und Ausscheiden aus dem Beruf wird teilweise erklärt durch einen hohen finanziellen Status, mit dem man es sich leisten kann, frühzeitig in den Ruhestand überzutreten, und der gleichzeitig ein höheres Gesundheitsniveau vorhersagt. Das Bildungsniveau hängt in ähnlicher Weise wiederum zusammen mit dem Gesundheitsstatus und den finanziellen Ressourcen.

In Bezug auf Geschlechtereffekte zeigt sich, dass Frauen im Durchschnitt häufiger als Männer frühzeitig in den Ruhestand übergehen. Dieser Effekt ist dann stärker oder besteht in einzelnen Studien nur dann, wenn ein geringerer Bildungsstand der Befragten vorliegt, die Frauen verheiratet bzw. nicht alleinstehend sind, wenn 
der/die Lebenspartner/in sich bereits im Ruhestand befindet, die Paarbeziehung als positiv beschrieben wird oder/und wenn eine Betreuungs- oder Pflegeaufgabe von Angehörigen im Ruhestand übernommen wird (Denton und Spencer 2009). Eine weitere Rolle spielt die persönliche Einstellung gegenüber dem Ruhestand. Eine negative Einstellung führt nicht nur dazu, dass man die Entscheidung bezüglich des Übergangs verzögert, sondern auch dazu, dass man den Ruhestand im Vorfeld weniger thematisiert und weniger gut plant.

\section{Der Ruhestandsprozess als Herausforderung}

Nun stellt sich der Frage, warum der Prozess des Ruhestands und insbesondere die Anpassung an den neuen Lebensabschnitt Herausforderungen darstellen können. Der Übergang in den Ruhestand gilt zunächst als neutrales Ereignis, als Entwicklungsaufgabe, die im Lebenslauf verankert ist. Der Übergang kann sich jedoch zum kritischen Lebensereignis entwickeln (Barbosa et al. 2016; Hübner 2016; Luhmann et al. 2012). Kritische Lebensereignisse können als anregend und zugleich belastend empfunden werden, z.B. weil sie für den Betroffenen mit Kosten verbunden sind, gleichzeitig aber auch Nutzen mit sich bringen.

Der Übergang in den Ruhestand als bedeutsamer Einschnitt im Leben eines Menschen kann mit Verlusten einhergehen, die durch die Deprivation manifester und latenter bzw. psychosozialer Funktionen der Arbeit entstehen (Jahoda 1983). Eine zentrale manifeste Funktion der Arbeit ist die finanzielle Entlohnung oder auch finanzielle Sicherheit. Durch den Übergang in den Ruhestand sinkt in der Regel das Einkommen bzw. die ökonomische Situation verändert sich, was zu Ängsten und zu Unsicherheit führen und negative gesundheitliche Konsequenzen haben kann. Der Übergang in den Ruhestand hat jedoch nicht nur ökonomische Folgen, sondern es kommt, ähnlich wie bei Arbeitslosigkeit, zu einem Wegfall sogenannter latenter oder psychosozialer Funktionen (Jahoda 1983; Wang et al. 2011). So ist Arbeit z.B. ein Ort für sozialen Kontakt, Austausch und Kooperation. Arbeit strukturiert den Tagesablauf, zwingt zu Aktivität und definiert die zu erreichenden Ziele. In der Arbeit kann man vielfältige Aufgaben meistern und eigene Handlungskompetenz zum Ausdruck bringen, wofür man Anerkennung erhält. Durch den Ruhestand entfällt eine jahrelang gewohnte Tätigkeit. Neue Tätigkeitsfelder müssen gefunden werden, die alltägliche Zeitorganisation und -struktur muss neugestaltet werden. Durch den Verlust der beruflichen Rolle entfällt zudem ein wichtiger Baustein der individuellen Identität, sodass Neugewichtungen von Rollen notwendig werden (z.B. eine Stärkung von familienbezogenen Rollen). Der Verlust der beruflichen Rolle kann mit Anpassungsschwierigkeiten verbunden sein. Demgegenüber werden Rollenübergänge beim Ruhestandsprozess von Personen mit einer hohen Eingebundenheit in andere nicht-berufliche Rollen bzw. mit einer geringen Identifizierung mit der Arbeitsrolle tendenziell weniger negativ wahrgenommen. Sie entscheiden sich tendenziell auch eher für einen zeitlich früheren Ausstieg aus dem Erwerbsleben.

Mit dem Ruhestand sind jedoch in der Regel auch vielfältige Gewinne verbunden. Diese wurden in der psychologischen Literatur der letzten Jahre zunehmend thematisiert. Ein wichtiger positiver Aspekt ist dabei die Zunahme der zeitlichen 
Flexibilität. Mehr zeitliche Ressourcen stehen für außer-berufliche Aktivitäten zur Verfügung, für Familie, Freunde, gesellschaftliches Engagement bei gleichzeitig geringerem Belastungserleben. Rentner, die während ihres Erwerbslebens nur wenig Selbstbestimmung erlebt haben und hohen Arbeitsbelastungen ausgesetzt waren, profitieren tendenziell stärker von der Zunahme an Flexibilität, Autonomie und Einflussnahme. Menschen unterscheiden sich darin, wie gut ihnen die Anpassung an den Ruhestand als neue Lebensphase gelingt. Außerdem kann die Anpassung sich mit der Zeit verändern: So kann sich das Wohlbefinden bei einem „Honeymoon“Effekt zunächst positiv entwickeln, man genießt die neue Lebensphase und freut sich darüber, endlich mehr Zeit für Familie und Freizeit zu haben. Nach einiger Zeit können sich jedoch die Effekte der Deprivation manifester und latenter Faktoren bemerkbar machen, Leere und Frustration können entstehen. Daneben gibt es auch umgekehrte Verläufe, bei denen das Wohlbefinden zunächst abnimmt, man sich nach einiger Zeit im Ruhestand aber erholt und gut an die neue Lebensphase anpasst (Pinquart und Schindler 2007; Wang 2007).

In welche Richtung die Entwicklung des Einzelnen ausschlägt, wie sich der Verlauf in der Ausprägung von Gewinnen und Verlusten im Ruhestandsprozess gestaltet und wie in der Konsequenz die Anpassung über die Zeit variiert, ist wesentlich abhängig von vielfältigen persönlichen Ressourcen. Wang et al. (2011) haben das Ressourcen-basierte dynamische Modell des Ruhestandprozesses entwickelt, um Anpassungsverläufe an den Ruhestand sowie die potenzielle Entstehung von Problemen im Übergang abzubilden und darüber hinaus interindividuelle Unterschiede in der Anpassung über die Zeit zu erklären. Eine zentrale Idee ist, dass der Ruhestandsprozess von bestimmten persönlichen Ressourcen abhängt. Ressourcen werden dabei recht breit formuliert als Objekte, Eigenschaften, Bedingungen, die vom Individuum geschätzt werden und als Mittel zum Erreichen persönlicher Ziele dienen (Halbesleben et al. 2014; Hobfoll et al. 2018). Ressourcen erleichtern den Umgang mit der neuen Lebenssituation im Ruhestand und sind damit einer positiven Entwicklung zuträglich. Nach Hobfoll (1989) sind Menschen bestrebt, Ressourcen zu schützen, zu erhalten und neue Ressourcen aufzubauen. Je mehr Ressourcen vorhanden sind, desto besser gelingt der Anpassungsprozess (Hobfoll et al. 2018).

Im Allgemeinen werden interne Ressourcen (Eigenschaften, Kompetenzen, Fähigkeiten wie z.B. Bewältigungsstrategien im Umgang mit schwierigen Lebenssituationen) abgegrenzt von externen Ressourcen (durch die Umwelt bedingte Einflüsse, z. B. soziale Unterstützung). Wang et al. (2011) unterscheiden ferner zwischen verschiedenen Ressourcenkategorien: emotionale Ressourcen, z. B. eine generelle und situationsunabhängige positive Stimmungslage, gute Emotionskontrolle und die Kompetenz, Misserfolge zu bewältigen; kognitive Ressourcen, z. B. Optimismus, Selbstwirksamkeit sowie eine ausgeprägte kognitive Funktionsfähigkeit (u.a. Gedächtnisleistung, Informationsverarbeitungskapazität); motivationale Ressourcen oder das Ausmaß von Engagement und Persistenz im Verfolgen seiner Ziele; soziale Ressourcen, Unterstützung durch Andere und soziale Eingebundenheit; physische Ressourcen, die wahrgenommene körperliche Gesundheit, und schließlich finanzielle Ressourcen. Die individuelle Ausprägung dieser Ressourcen wird wiederum determiniert durch Eigenschaften der Person selbst (u. a. Geschlecht, Familienstand, 
Bildungsniveau) und durch kontextuelle Faktoren wie die Verfügbarkeit von sozialen Sicherungssystemen in einer Gesellschaft.

\section{Determinanten der Anpassung an den Ruhestand}

Um Aussagen über die Entwicklung und Veränderung in der Anpassung an den Ruhestand treffen zu können, sind empirische Längsschnittstudien notwendig. Wang (2007) hat insgesamt mehr als 1900 Personen aus den USA über einen Zeitraum von acht Jahren in Interviews zu ihrem emotionalen Wohlbefinden befragt. Die Personen wurden vor dem Übergang in den Ruhestand sowie wiederholt während des Ruhestands im Rahmen von Haushaltsbefragungen kontaktiert (vgl. Pinquart und Schindler 2007).

Die Ergebnisse zeigen, dass $70 \%$ der Befragten nur geringfügige Veränderungen im Wohlbefinden nach dem Übergang in den Ruhestand berichten (Erhaltungsverlauf). Dies trifft für Personen zu, die auch im Ruhestand einer beruflichen bzw. vergüteten Tätigkeit nachgehen (z.B. in der Form einer geringfügigen Beschäftigung). Die Tatsache, weiterhin beruflich aktiv sein zu können, hängt eng zusammen mit einem guten Gesundheitsstatus: Nur solche Ruheständler mit guter Gesundheit können weiterhin einer beruflichen Tätigkeit nachgehen; gleichzeitig verbessert oder zumindest erhält aktives berufliches Engagement die Gesundheit. Weitere Forschung zeigt, dass sich die günstigen Effekte einer entlohnten Beschäftigung nur dann oder insbesondere dann ergeben, wenn der Übergang in den Ruhestand unfreiwillig war und finanzielle Aspekte der Tätigkeit nicht als primäres Motiv fungieren, sondern andere Motive wie der Wunsch, sich einzubringen und Wissen weiterzugeben, im Vordergrund stehen (Wang 2012; Wang und Shi 2014). Zudem zeigen Studien, dass neben der entlohnten Beschäftigung auch bürgerschaftliches Engagement oder ähnliche Aktivitäten mit positiven Konsequenzen für das individuelle Wohlbefinden einhergehen, wenn diese als für das Individuum bedeutungsvoll wahrgenommen werden können. Solche Formen des beruflichen und nicht-beruflichen Engagements bieten die Möglichkeit des Erhalts der psychosozialen Funktionen, die mit Arbeit einhergehen (u.a. Strukturierung des Tagesablaufs, Aufrechterhaltung von sozialen Kontakten). Auch kann eine umfassende Planung des Ruhestands die Stabilität im Wohlbefinden nach dem Übergang in den Ruhestand positiv vorhersagen (Wang 2007). Es geht dabei um die konkrete, sowohl instrumentelle als auch kognitive Vorbereitung auf den Ruhestand, nicht nur in Bezug auf finanzielle Aspekte, sondern auch in Bezug auf die inhaltliche Ausgestaltung des Ruhestands. Positive Erwartungen können Antrieb für Pläne im Ruhestand und eine aktive Gestaltung des neuen Alltags sein.

Die Studie von Wang (2007) zeigt zudem, dass bei $25 \%$ der Befragten ein geringerer Ausgangswert im Wohlbefinden bereits bei Eintritt in den Ruhestand vorliegt; sie zeigen in der Folge aber eine positive Entwicklung im Wohlbefinden, können sich also über die Zeit erholen (Erholungsverlauf). Dies trifft insbesondere auf Menschen zu, die zuvor einer körperlich und oder mental sehr belastenden beruflichen Tätigkeit nachgingen oder generell nur wenig zufrieden in und mit ihrer Arbeit waren. Bei einzelnen Personen (5\% in der Studie von Wang 2007) kommt es direkt 
nach dem Übergang in den Ruhestand zunächst zu einem Rückgang im emotionalen Wohlbefinden, der gefolgt ist von einer späteren Zunahme. Ein solcher U-förmiger Verlauf ergibt sich tendenziell bei Personen, die unerwartet bzw. früher als geplant und damit häufig auch unfreiwillig in den Ruhestand übergehen. Die Freiwilligkeit und die Terminierung des Übergangs spielen also eine wesentliche Rolle für die Anpassung insbesondere in den ersten Jahren nach erfolgtem Übergang. Wenn die Verrentung z.B. aufgrund längere Krankheit deutlich früher erfolgen muss, als es die gesetzliche Vorgabe vorsieht, kann dies negative Konsequenzen für das Wohlbefinden der betroffenen Personen haben. Ähnlich negative Effekte auf die Anpassung zeigen sich auch dann, wenn die Verrentung deutlich später als vorgesehen oder erwünscht erfolgt, z. B. aus finanziellen Gründen weiterhin beruflich aktiv sein zu müssen. In einer weiteren umfassenden längsschnittlichen Studien zeigt sich, dass Personen, die direkt aus einer Phase der Arbeitslosigkeit in den Ruhestand übergehen, eine sinkendende Lebenszufriedenheit über die Zeit im Ruhestand hinweg aufweisen (Pinquart und Schindler 2007).

Auch Personen mit gesundheitlichen Einschränkungen folgen direkt nach dem Übergang in den Ruhestand eher einem U-förmigen Verlauf. Auch solche Personen, die sich in einer unglücklichen Partnerschaftsbeziehung befinden, zeigen zunächst eine Reduktion im Wohlbefinden mit späterem Anstieg (vermutlich aufgrund fehlender sozialer Unterstützung). Weitere Längsschnittstudien verweisen zudem auf die Rolle des finanziellen Status im Ruhestand (Wang und Shi 2014). Das Ausmaß finanzieller Ressourcen determiniert mitunter die Möglichkeiten und Grenzen dessen, was im Ruhestand realisiert werden kann, inwiefern man seine Pläne umsetzen kann und Gestaltungsräume wahrnehmen und nutzen kann (Hübner 2016). Die finanzielle Situation hängt wiederum mit dem Bildungsniveau und dem individuellen Gesundheitszustand zusammen.

Erste empirische Belege gibt es zudem für die Rolle von Persönlichkeits- und psychologischen Eigenschaften in der Vorhersage der Anpassung an den Ruhestand. Eine generell positive Einstellung gegenüber der eigenen Person und der Umwelt, ein fester Glaube an die eigene Kompetenz und Leistung sowie eine hohe emotionale Ausgeglichenheit erweisen sich als förderlich (Barbosa et al. 2016). Gleichzeitig dürfen auch kontextuelle Ressourcen nicht unberücksichtigt bleiben. Relevant in Bezug auf die Anpassung sind Faktoren auf gesellschaftlicher und staatlicher Ebene (u. a. Altersbilder und gesellschaftliche Altersstereotype, Lebensqualität des Landes, in dem man in den Ruhestand übergeht, finanzielle und institutionelle Unterstützung von Seiten des Staates) (Hübner 2016; Wang und Shi 2014).

\section{Diskussion und Schlussfolgerungen}

Insgesamt kommen bisherige empirische Studien also zu dem Ergebnis, dass der Verlauf der Anpassung an den Ruhestand bei der Mehrzahl der befragten Personen über die Zeit hinweg tendenziell positiv ist (Luhmann et al. 2012; Pinquart und Schindler 2007; Wang 2007). Jedoch erleben einige Personen den Ruhestand als krisenhaftes Ereignis, was zu einer problematischen Entwicklung führen kann. In Anlehnung an den hier dargestellten Ressourcen-basierten dynamischen Ansatz des 
Ruhestandprozesses können Unterschiede zwischen Menschen durch individuelle und kontextuelle, arbeitsbezogene Ressourcen sowie deren Zusammenspiel erklärt werden, von denen einige im Rahmen von längsschnittlichen Studien empirisch getestet wurden. Davon lassen sich praktische Implikationen ableiten.

Eine frühzeitige Vorbereitung und konkrete Auseinandersetzung mit der Zeit nach der Erwerbstätigkeit kann eine erfolgreiche Anpassung fördern und dazu beitragen, dass der Ruhestand als weniger einschneidend und bedrohlich für das individuelle Wohlbefinden wahrgenommen wird. Die Planung des Ruhestands findet eher statt, wenn eine positive Erwartungshaltung und Einstellung gegenüber dem Ruhestand besteht. Das Entwickeln von Szenarien für die Zeit nach dem Ausscheiden aus dem Beruf sollte möglichst konkret erfolgen und mit nahestehenden Menschen besprochen werden. Welche Ziele hat man für den Ruhestand? Was kann man schon im Vorfeld tun, um diese Ziele zu realisieren? Was wären alternative Ziele und ein Plan B?

Vorhandene Studien zeigen, dass Menschen im Ruhestand in der Regel gerne weiterhin aktiv und eingebunden sein möchten. Die Möglichkeit, einer sinnhaften und ausfüllenden Tätigkeit nachzugehen, wirkt sich positiv auf den Anpassungsverlauf aus (Wang und Shi 2014). Eine zentrale Funktion des Eingebunden-Seins in entlohnte oder nicht entlohnte Tätigkeiten im Ruhestand ist die Aufrechterhaltung der Handlungskompetenz und der Strukturierung des Alltags. Um welche Tätigkeiten und Aktivitäten es sich dabei handelt (u. a. Formen der Weiterbeschäftigung, geringfügige Beschäftigung, ehrenamtliches Engagement etc.), ist weniger relevant als die Frage, was als individuell passend und möglichst zufriedenstellend wahrgenommen wird.

Bisher gibt es nur wenige evidenzbasierte und systematische praktische Ansätze und Interventionen zur Vorbereitung auf den Ruhestand, die die psychologische Perspektive integrieren. Bestehende Programme fokussieren meist auf finanzielle Fragen bzw. die finanzielle Planung des Ruhestands. Einen vielversprechenden Ansatz liefert das Programm der Ressourcenaktivierung nach Seiferling und Michel (2017), das auf Basis des Ressourcen-basierten Modells zur optimalen Vorbereitung auf den Ruhestand entwickelt wurde. Der Interventionsansatz zielt darauf ab, bereits in der Zeit vor dem Ruhestand individuelle psychologische Ressourcen (u.a. Optimismus, Selbstwirksamkeit, individuelle Stärken und Kompetenzen, Ziele für die Zeit im Ruhestand), soziale Ressourcen (soziales Netzwerk) und materielle Ressourcen (finanzielle Situation) zu aktivieren, die einen optimalen Übergang und eine erfolgreiche Anpassung auf Basis empirischer Erkenntnisse fördern. Gleichzeitig geht es auch darum, konkrete Ziele für den Ruhestand zu entwickeln, und es wird herausgearbeitet, wie Ziele bestmöglich verfolgt werden können, welche Hürden und Barrieren auftauchen können, die die Zielerreichung erschweren, und wie eine Abkehr von Zielen gelingen kann, deren Erreichbarkeit gefährdet oder unmöglich ist.

Neben Individuums-bezogenen Ansätzen sollten auch Maßnahmen auf der Makroebene (u. a. Gestaltung von Rentensystemen, gesetzliche Regelungen) und auf der Ebene der Unternehmen die psychologische Perspektive stärker berücksichtigen, um Menschen beim Übergang und im Verlauf des Ruhestands zu unterstützen. Auf organisationaler Ebene gibt es einzelne nennenswerte Ansätze wie Lebensphasen- und 
Alters-orientierte Personalentwicklungsstrategien. Diese forcieren Arbeitsmodelle zur Förderung eines fließenden Übergangs in den Ruhestand, umfassen konkrete Unterstützung und Beratung im Vorfeld des Übergangs und sehen auch Möglichkeiten zum aktiven Einbezug von Ruheständlern in die Organisation vor (z. B. im Rahmen von Mentoring-Funktionen) (Deller und Kolb 2010; Gerlmaier et al. 2016; Hübner 2016).

Insgesamt gibt es in Bezug auf den Übergang und die erfolgreiche Anpassung an den Ruhestand an der Schnittstelle zwischen Theorie und Praxis großen Handlungsbedarf. Außerdem ist auch weitere empirische Forschung notwendig. Psychologische Forschung sollte sich dabei auf verschiedene Indikatoren der Anpassung an den Ruhestand fokussieren und das Zusammenspiel vielfältiger individueller und kontextueller Ressourcen im längeren Zeitverlauf systematisch untersuchen.

Open Access Dieser Artikel wird unter der Creative Commons Namensnennung 4.0 International Lizenz (http://creativecommons.org/licenses/by/4.0/deed.de) veröffentlicht, welche die Nutzung, Vervielfältigung, Bearbeitung, Verbreitung und Wiedergabe in jeglichem Medium und Format erlaubt, sofern Sie den/die ursprünglichen Autor(en) und die Quelle ordnungsgemäß nennen, einen Link zur Creative Commons Lizenz beifügen und angeben, ob Änderungen vorgenommen wurden.

\section{Literatur}

Baltes, P. B., \& Baltes, M. M. (1990). Psychological perspectives on successful aging: the model of selective optimization with compensation. In P. B. Baltes \& M. M. Baltes (Hrsg.), Successful aging: perspectives from the behavioral sciences (S. 1-34). New York: Cambridge University Press.

Barbosa, L. M., Monteiro, B., \& Murta, S.G. (2016). Retirement adjustment predictors-A systematic review. Work, Aging and Retirement, 2(2), 262-280.

Bliese, P., Adler, A. B., \& Flynn, P. J. (2017). Transition processes: a review and synthesis integrating methods and theory. Annual Review of Organizational Psychology and Organizational Behavior, 4 , 263-286.

Brandtstädter, J., \& Renner, G. (1990). Tenacious goal pursuit and flexible goal adjustment: explication and age-related analysis of assimilative and accommodative strategies of coping. Psychology and Aging, 5,58 .

Deller, J., \& Kolb, P. (2010). Herausforderung Demografie und Wandel der Arbeitsgesellschaft. In B. Werkmann-Karcher \& J. Rietiker (Hrsg.), Angewandte Psychologie für das Human Resource Management: Konzepte und Instrumente für ein wirkungsvolles Personalmanagement (S. 421-433). Berlin, Heidelberg: Springer.

Denton, F. T., \& Spencer, B. G. (2009). What is retirement? A review and assessment of alternative concepts and measures. Canadian Journal on Aging, 28, 63-76.

de Wind, A., Geuskens, G. A., Reeuwijk, K.G., Westerman, M.J., Ybema, J.F., Burdorf, A., \& van der Beek, A.J. (2013). Pathways through which health influences early retirement: a qualitative study. BMC Public Health, 13, 292. https://doi.org/10.1186/1471-2458-13-292.

Fisher, G. G., Chaffee, D.S., \& Sonnega, A. (2016). Retirement timing: A review and recommendations for future research. Work, Aging and Retirement, , 2, 230-261.

Gerlmaier, A., Gül, K., Hellert, U., Kämpf, T., \& Latniak, E. (2016). Praxishandbuch lebensphasenorientiertes Personalmanagement: Fachkräftepotenziale in technischen Entwicklungsbereichen erschließen und fördern. Wiesbaden: Springer.

Halbesleben, J.R.B., Neveu, J.-P., Paustian-Underdahl, S.C., \& Westman, M. (2014). Getting to the "COR" understanding the role of resources in conservation of resources theory. Journal of Management, 40, 1334-1364.

Havighurst, R. J. (1961). Successful aging. The Gerontologist, 1, 8-13.

Heckhausen, J., Wrosch, C., \& Schulz, R. (2010). A motivational theory of life-span development. Psychological Review, 117, 32-60.

Hobfoll, S.E. (1989). Conservation of resources: A new attempt at conceptualizing stress. American Psychologist, 44, 513-524. 
Hobfoll, S. E., Halbesleben, J., Neveu, J.-P., \& Westman, M. (2018). Conservation of resources in the organizational context: The reality of resources and their consequences. Annual Review of Organizational Psychology and Organizational Behavior, 5, 103-128.

Hübner, I.-M. (2016). Subjektive Gesundheit und Wohlbefinden im Übergang in den Ruhestand: eine Studie über den Einfluss und die Bedeutsamkeit des subjektiven Alterns und der sozialen Beziehungen. Wiesbaden: Springer.

Jahoda, M. (1983). Wieviel Arbeit braucht der Mensch? Arbeit und Arbeitslosigkeit im 20. Jahrhundert. Weinheim: Beltz.

Lippke, S., \& Renneberg, B. (2006). Theorien und Modelle des Gesundheitsverhaltens. In B. Renneberg \& P. Hammelstein (Hrsg.), Gesundheitspsychologie (S. 35-60). Berlin: Springer.

Luhmann, M., Hofmann, W., Eid, M., \& Lucas, R. E. (2012). Subjective well-being and adaptation to life events: a meta-analysis. Journal of Personality and Social Psychology, 102, 592-615.

Pinquart, M., \& Schindler, I. (2007). Changes of life satisfaction in the transition to retirement: a latentclass approach. Psychology and Aging, 22, 442-455.

Seiferling, N., \& Michel, A. (2017). Building resources for retirement transition: Effects of a resourceoriented group intervention on retirement cognitions and emotions. Work, Aging and Retirement, 1-18. https://doi.org/10.1093/workar/wax011.

Shultz, K. S., \& Wang, M. (2011). Psychological perspectives on the changing nature of retirement. American Psychologist, 66, 170.

Statistisches Bundesamt (2006). Bevölkerung Deutschlands bis 2050. 11. koordinierte Bevölkerungsvorausberechnung. Wiesbaden: DeStatis.

Tesch-Römer, C., Engstler, H., \& Wurm, S. (2006). Altwerden in Deutschland: Sozialer Wandel und individuelle Entwicklung in der zweiten Lebenshälfte. Wiesbaden: VS.

Van Solinge, H., \& Henkens, K. (2008). Adjustment to and satisfaction with retirement: two of a kind? Psychology and Aging, 23, 422-434.

Wang, M. (2007). Profiling retirees in the retirement transition and adjustment process: examining the longitudinal change patterns of retirees' psychological well-being. Journal of Applied Psychology, $92,455-474$.

Wang, M. (2012). The Oxford handbook of retirement. New York: Oxford University Press.

Wang, M., \& Shi, J. (2014). Psychological research on retirement. Annu. Rev. Psychol, 65, 209-233.

Wang, M., Henkens, K., \& van Solinge, H. (2011). Retirement adjustment: A review of theoretical and empirical advancements. American Psychologist, 66, 204.

WHO (2017). Life expectancy. http://www.who.int/gho/mortality_burden_disease/life_tables/situation_ trends_text/en/. Zugegriffen: 15. Nov. 2017.

Wöhrmann, A. M., Pundt, L., \& Deller, J. (2017). Silver Careers: Laufbahngestaltung im Ruhestand. In S. Kauffeld \& D. Spurk (Hrsg.), Handbuch Karriere und Laufbahnmanagement (S. 1-22). Berlin: Springer.

Zacher, H. (2015). Successful aging at work. Work, Aging and Retirement, 1, 4-25.

Zacher, H. (2017). Berufliche Veränderungen: Wenn Erwerbstätige sich neu orientieren. In S. Kauffeld \& D. Spurk (Hrsg.), Handbuch Laufbahnmanagement und Karriereplanung. Berlin: Springer.

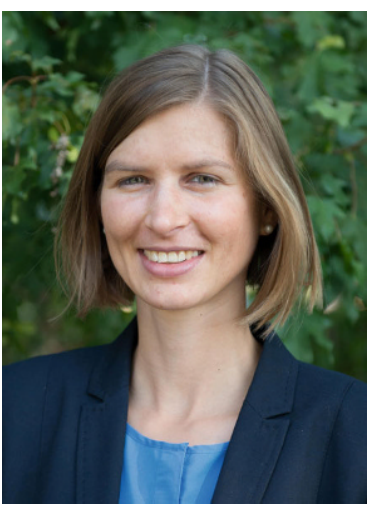

Dr. Antje Schmitt Dipl.-Psych., Systemische Beraterin (SG), Assistenzprofessorin an der Universität Groningen (Niederlande), Fachgebiet Organizational Psychology. Tätigkeitsschwerpunkte: Forschung und Lehre zu Wohlbefinden im Arbeitskontext und proaktivem Arbeitshandeln. 